\title{
DESIGN OF FAST MAGNETORHEOLOGICAL DAMPER USING SELECTIVE LASER MELTING TECHNOLOGY
}

\author{
P. Vítek, Z. Strecker*, J. Roupec
}

\begin{abstract}
This paper examines a potential of structured magnetic circuits for a construction of piston of magnetorheological (MR) damper with short response time, great force range and good mechanical properties. Using complex structures designed according to the magnetic FEM simulation can almost eliminate eddy-currents (and thus response time), reduce the weight of magnetic circuit with only a small drop in magnetic flux in the magnetic circuit. Due to the complexity of shapes of structured magnetic circuits, selective laser melting (SLM) technology is currently considered as the only possible technology. A shape optimization with respect to SLM limitations is therefore proposed. As a material for the magnetic circuit, nearly pure Fe was chosen. This material has very good magnetic properties but the drawback is a very good electric conductivity, which causes large generation of eddy currents during rapid changes of magnetic field. Suggested structured magnetic circuit is compared to a solid magnetic circuit by means of magnetic simulations. Simulations showed that the structured geometry of magnetic circuits can effectively reduce its weight (the weight of bobbin was reduced by up to $45 \%$ and the weight of outer cylinder was reduced by up to $13 \%$ ) while time response is reduced more than 18 times and inductance drop is less than $20 \%$.
\end{abstract}

Keywords: MR damper, selective laser melting, structured geometry, time response

\section{Introduction}

There are two very important parameters that need to be taken into account when designing magnetorheological damper - a response time and a dynamic range. The influence of response time on efficiency of semiactive car suspension with MR damper was studied by Strecker (2015). He found out that for effective Modified Groundhook algorithm control of passenger car suspension, the response time must be lower than $1.5 \mathrm{~ms}$. Goncalves (2005) measured response time of the MR fluid which is around $0.6 \mathrm{~ms}$. The long response time of the MR damper is caused mostly by the induction of eddy currents in the magnetic circuit during rapid changes of electric current to the coil. There are two ways how to eliminate eddy currents. The first option is to use a material with high electrical resistivity. Strecker (2015) tested this option using ferrite material Epcos N87. The best achieved response time of magnetic field (with full ferrite piston) was $0.31 \mathrm{~ms}$. A disadvantage of this material is a low magnetic saturation $(490 \mathrm{mT})$ which causes low dynamic range, bad mechanical properties and poor machinability. The second option is a shape optimization. Strmiska (2017) designed a MR piston made of free-cutting steel 11SMn30 with magnetic saturation 1.9 T. The bobbin and outer cylinder had radial grooves along the circumference. With this geometry, the response time was shortened to $0.5 \mathrm{~ms}$. The magnetic flux density compared to solid version was only $10 \%$. Another possibility is a use of additive technology, e.g., Selective laser melting (SLM). A big advantage is a greater freedom in terms of designing the structure of magnetic circuit, however, the portfolio of processed materials is still very limited. The SLM technology also allows weight minimization by targeted material removal. One of suitable materials appears to be Fe-Ni alloy. Manufacturing on SLM and resulting magnetic properties were studied by Zhang (2012). The magnetic properties were similar to properties of alloy produced by mechanical alloying. Another promising material is nearly pure iron. Permeability and magnetic saturation of this material are very high. Its process parameters were studied by Palousek (2017).

All authors: Institute of Machine and Industrial Design, Brno Technical University, Technická 2896/2; 616 69, Brno; CZ, strecker@fme.vutbr.cz 


\section{Methods}

The main objective was a design a MR damper piston with good mechanical properties, short response time of magnetic field, high maximum magnetic flux density in the gap and low weight. In order to keep small coil and low electric currents, the magnetic resistivity of the magnetic circuit must be as low as possible. On the other hand, in order to keep response time as low as possible, it is necessary to avoid generation of eddy currents by high electric resistivity. These two demands can be fulfilled by material or shape approach. We have used the shape approach.

The eddy currents are always generated in the perpendicular plane to the magnetic flux lines (Fig. 1b). Therefore, if the magnetic circuit is manufactured from set of beams which follow magnetic flux lines and which are electrically isolated from each other, the electric resistance for eddy currents will be very high and the eddy currents will be significantly reduced. This arrangement will also secure only a very small rise of magnetic resistance of magnetic circuit. The complex shape of magnetic circuit can be manufactured by SLM method from pure iron. Pure iron was chosen because of experience with this material on our workplace. This method also enables to keep constant cross sections of beams in all the parts of magnetic circuit, which secures the weight reduction of magnetic core with only a small negative impact on maximal magnetic flux density in the piston gap.

The geometry of the MR damper piston that was used for simulations is in Fig.1b and optimized segmented MR damper piston is in Fig. 1c. Parts that have been subjected to optimization were bobbin and outer cylinder.

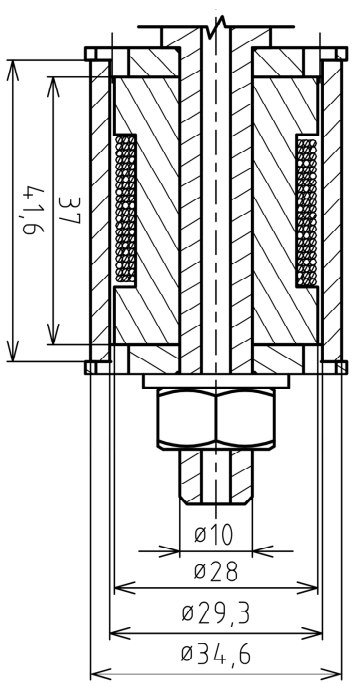

(a)

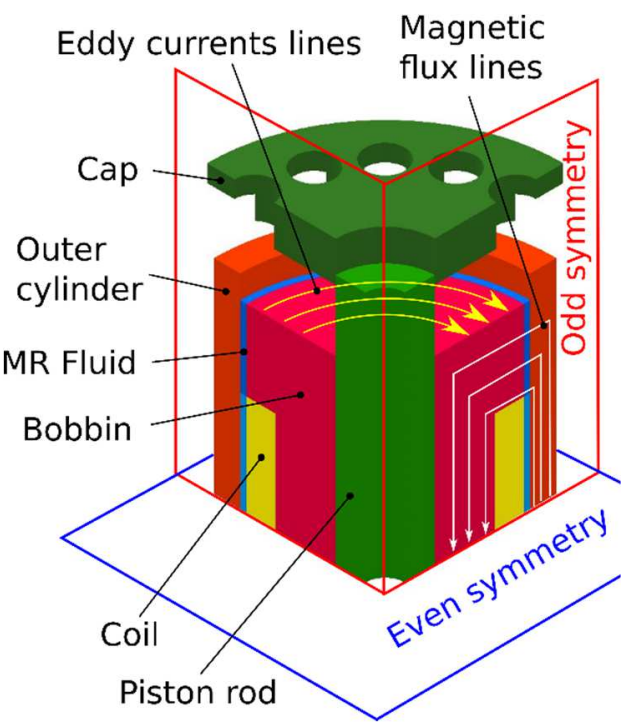

(b)

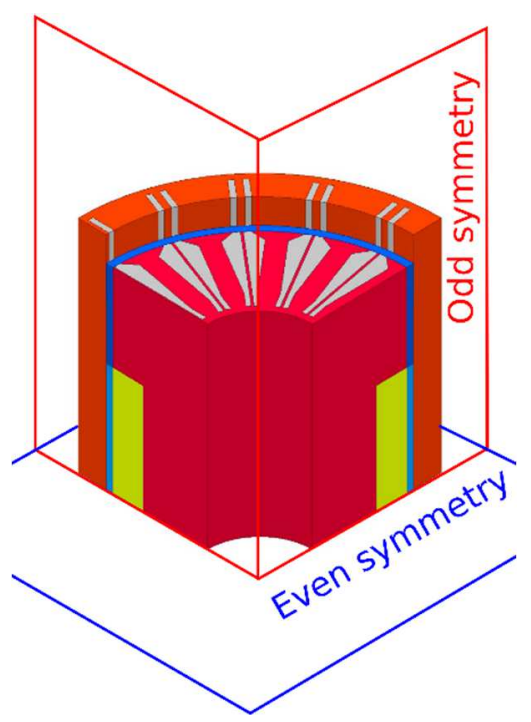

(c)

Fig. 1: MR piston configuration (a) dimensions, (b) configuration of 1/8 model for magnetic FEM simulation, (c) configuration of 1/8 model of optimized piston for magnetic FEM simulation

\subsection{Magnetic model}

In order to verify the proposed shape optimization, a magnetic model was created in Ansys electronics 17.1. Due to the nature of the task, the simulation model was performed in 3D. Thanks to the geometrical symmetry of the MR piston, the simulation model has been simplified to $1 / 8$ as is shown in Fig. 1. The gap was filled by MR fluid LORD MRF132DG.Piston rod is made of S235JRG. The winding consisted of 120 turns. Two types of simulations were performed. Static simulations to determine B-I characteristics and transient simulations to determine response of magnetic flux density in the gap. The time step of transient analysis was time dependent and varied from 0.05 to $2 \mathrm{~ms}$, the length based mesh consisted of 29892 elements in case of solid MR piston and 213372 elements in case of geometrically optimized MR piston. The static simulations were done for currents between $0-5 \mathrm{~A}$. The transient simulations were done for current decrease from 2 A to 0 A during $0.3 \mathrm{~ms}$.

\subsection{The method of determining the time response}

The response time was evaluated from the course of magnetic flux density in the gap as a response on $0.3 \mathrm{~ms}$ linear drop of electric current in the coil. The time needed for dropping to $36.8 \%$ of a steady state magnetic flux density for initial current was considered as time response. 


\subsection{Optimized geometry}

In Fig. 2, there is a visualization of optimized geometry of bobbin and outer cylinder. The designed structure consists of beams, that follows paths of magnetic flux in the solid version of the magnetic circuit in steady state that are shown in Fig. 1 (b). The beams have constant cross-sectional area in almost the whole length.

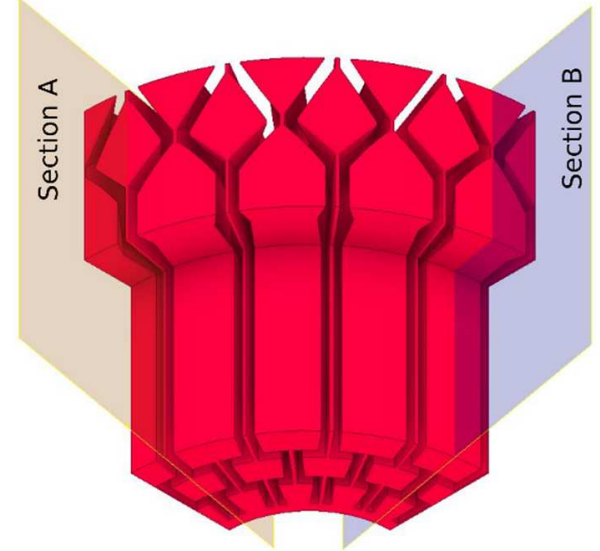

(a)

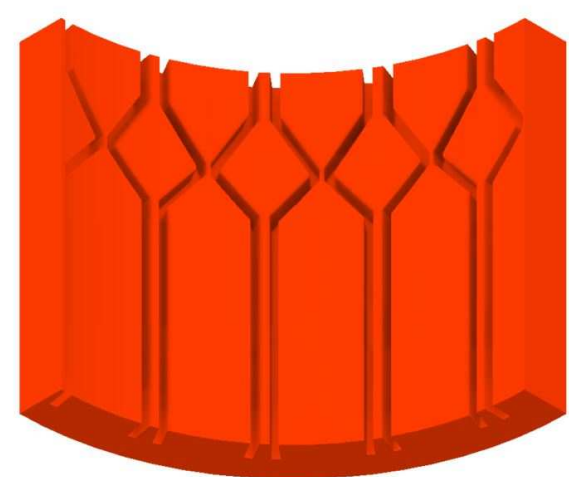

(b)

Fig. 2: Optimized segmented piston respecting the magnetic flux paths of (a) bobbin, (b) outer cylinder

\section{Results}

Fig. 3 shows magnetic flux density in a central beam. Even though the cross-section of the beam in bobbin is kept constant, the magnetic flux density in radial part of the beam is significantly higher. This effect is caused mostly because part of the magnetic flux is conducted by piston rod. The piston rod is practically increasing the effective cross-section of magnetic flux path.

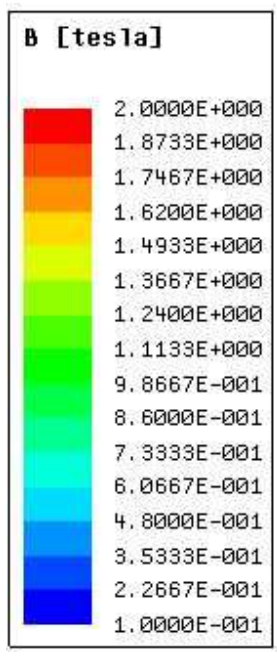

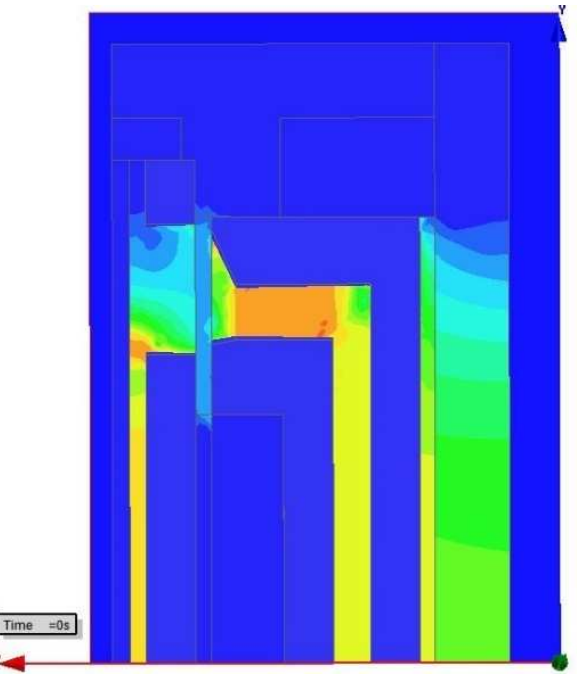

(a)

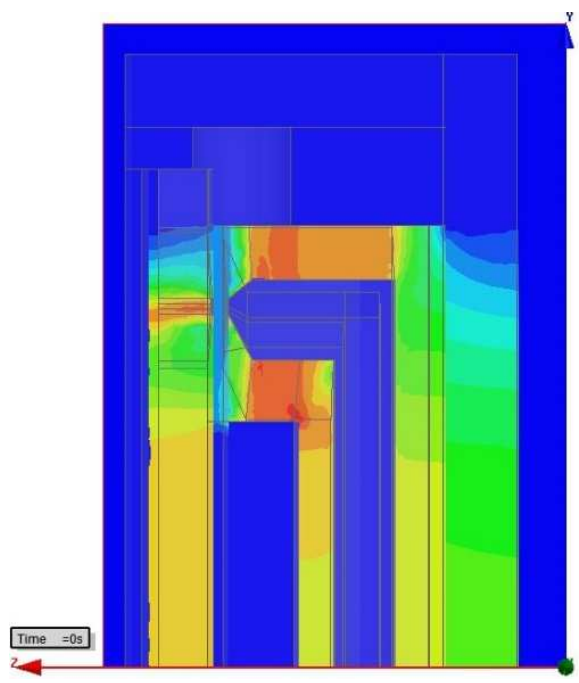

(b)

Fig. 3: Magnetic flux density in (a) central beam (section A), (b) outer beams (section B)

\subsection{Static and transient simulations}

Fig. 4a compares magnetic flux densities in gap of solid and structured version of the magnetic circuit. The figure implies that the optimized magnetic circuit reaches around $20 \%$ lower magnetic induction in the gap. It is due to the earlier magnetic saturation of bobbin caused mostly by the smaller cross-section. The isolation distance between beams is $0.5 \mathrm{~mm}$ which respects the smallest manufacturable distance on the SLM printer SLM 280HL. Fig.4b compares response times of magnetic flux density on the electric current of pistons with solid and structured magnetic circuit in the gap. Time response of the solid piston is $4.4 \mathrm{~ms}$ whereas time response of structured piston is $0.32 \mathrm{~ms}$. 


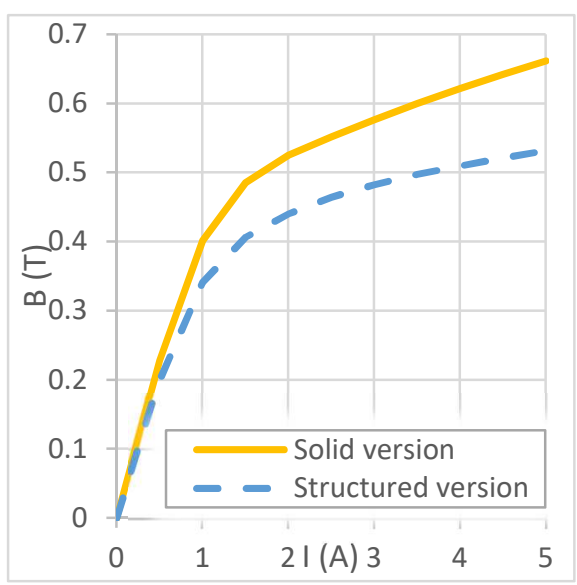

(a)

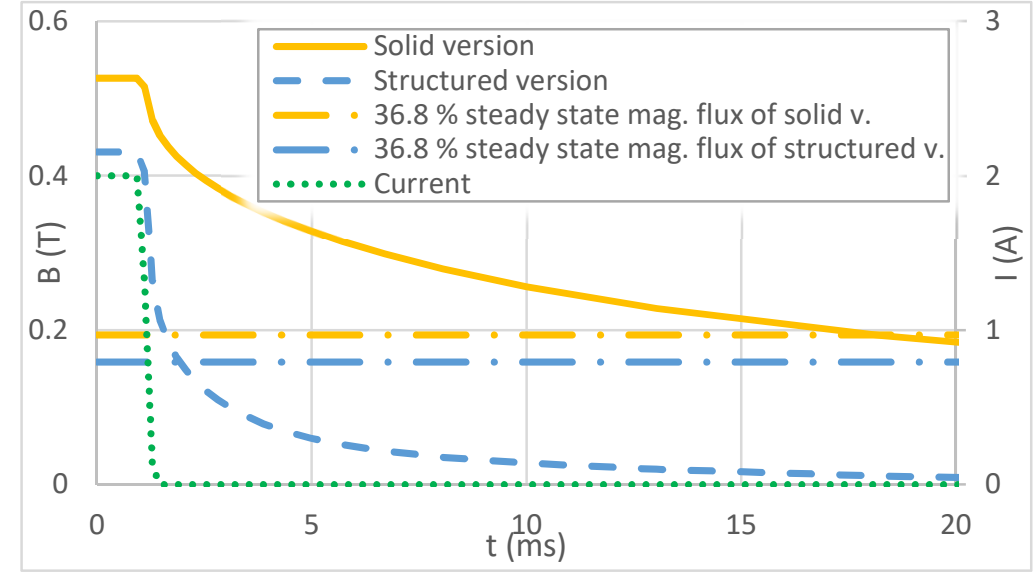

(b)

Fig. 4: Comparison of solid and structured piston through (a) static, (b) transient simulation

Tab. 1: Performance comparison of pistons

\begin{tabular}{cccc}
\hline Type of piston & Time response (ms) & Magnetic flux (T) & Weight (g) \\
\hline Solid piston & 17.12 & 0.526 & 201 \\
\hline Segmented piston & 0.93 & 0.431 & 138 \\
\hline
\end{tabular}

\section{Conclusions}

This paper deals with the new design of magnetic circuit of MR damper, which benefits from possibilities of SLM technology. The SLM technology enables to print magnetic circuits from pure iron or steel with good mechanical properties. Special structure eliminating eddy currents secures very short response time of magnetic flux density in the gap, low weight and high magnetic flux density in the gap. The proposed geometry decreases weight of the bobbin by $45 \%$ and by $13 \%$ of weight of outer cylinder. The inductance drop of the magnetic circuit is less than $20 \%$ and time response is shortened more than 18 times in comparison with solid piston. The response time can be shortened more than two times if soft magnetic steel, e.g. 11SMn30 is used. The magnetic flux density can be increased by smaller isolation. The only ferromagnetic material which can be printed on SLM 280HL printer, which is present in our department and which will be used for printing of magnetic circuit, is at the moment unfortunately only pure iron.

\section{Acknowledgement}

This experiment could be provided thanks to the kind sponsorship of various grants and numerous agencies. We would like to explicitly thank to the GAČR 17-10660J, GAČR 17-26162S, FSI-S-17-4428.

\section{References}

Goncalves, F.D., Ahmadian, M. and Carlson, J.D. (2005) Investigating the magnetorheological effect at high flow velocities. Smart Materials and Structures, 15, 1, pp. 75-85.

Palousek, D., Pantelejev, L., Zikmund, T., et al. (2017) Processing of nearly pure iron using 400W selective laser melting - initial study. MM Science Journal: 1738-1743.

Strecker, Z., Roupec, J., Mazurek, I., et al. (2015) Design of magnetorheological damper with short time response, Journal of Intelligent Material Systems and Structures, 26, 14, pp. 1951-1958.

Strecker, Z., Mazůrek, I., Roupec, J., et al. (2015) Influence of MR damper response time on semiactive suspension control efficiency, Meccanica, 50, 8, pp. 1949-1959.

Strmiska, T. (2017). The design of fast magnetorheological valves without the use of ferrites. Master's thesis. Brno University of Technology.

Zhang, B., Fenineche, N.E., Zhu, L., Liao and Coddet, C. (2012) Studies of magnetic properties of permalloy (Fe$30 \% \mathrm{Ni}$ ) prepared by SLM technology. Journal of Magnetism and Magnetic Materials, 324, 4, pp. 495-500.D SLM printer 